\title{
Natural Computing Paradigm - A Concise Introduction
}

\author{
Takashi Yokomori \\ Department of Mathematics, Faculty of Education and Integrated Arts and Sciences, \\ Waseda University, Tokyo 169-8050, Japan \\ E-mail:yokomori@waseda.jp
}

\begin{abstract}
Natural computing (NC) is an emerging area of research that investigates computing techniques and models inspired by nature on one hand, and it also investigates phenomena taking place in nature in terms of computational methodologies on the other hand. Thus, research in NC congenitally has interdisciplinary flavor, which bridges between computer science and various disciplines of natural science. Because of its interdisciplinary nature, NC connects and covers a broad spectrum of fundamental research fields including biology, chemistry, physics, medical science, and so forth. In this article, we give a concise introduction to the new computing paradigm of NC. Specifically, we give an overview of selected topics of the fields from theory to experiments, where the stress is primarily put on theoretical achievements in computing paradigms called molecular computing and chemical reaction computing.
\end{abstract}

Keywords: Natural computing, molecular computing, self-assembly, chemical reaction computing.

\section{Introduction}

Natural computing (NC) is a novel computing paradigm inspired by nature in which $\mathrm{NC}$ concerns a variety of computational aspects and potential features found in natural phenomena. Also, $\mathrm{NC}$ has close relations to computational process inspired by those phenomena.

The goal of NC includes the following research themes. (i) From nature to information processing which means investigating computational mechanism and its implementation based on lessons from nature, and

(ii) From information processing to nature which means computational analysis of nature in terms of designing new computing models and computer experiments (simulations). Further, NC also intends to exploit new applications in the fields of various disciplines involved. It is rather surprising that the conceptual source of the idea of NC has already appeared in an article of Biofizika in 1973 where an idea of cell molecular computers was discussed. Since 1974 Conrad has been conducting consistent research on the information processing capability using macro-molecules (such as proteins), and has edited a special issue in a journal entitled Molecular Computing Paradigms. His initial efforts in the early days of molecular computing have been succeeded by Head's

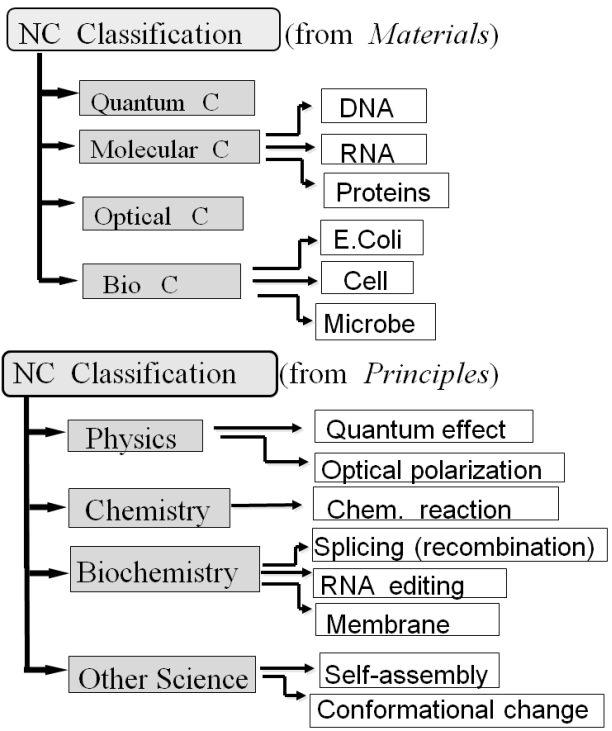

Figure 1. Natural Computing Paradigms Inspired by Nature (A part) 
original work on Splicing Systems and Languages whose theoretical results on splicing phenomena should be highly appreciated, in that it was the first achievement by means of mathematical analysis on bio-chemical operations of DNA recombination. It is possible to discuss about new computing paradigms inspired by nature with a general schema of "Computing with $X$ ", where $X$ ranges from various sorts of materials to those of principles. Figure 1 illustrates a part of natural computing paradigms proposed so far within the "Computing with $X$ " schema.

In this short article, among various computing paradigms we take up two computing paradigms "Molecular computing" (from materials), and "Chemical reaction computing" (from principles), and give an outline of fundamental results on computing capability of these new challenging ingenuity.

\section{Molecular Computing Paradigm}

In the early days, the goal of molecular computing seemed to replace silicon-based hardware with biomolecular one. Unlike this intention of the early schema, however, by now it is commonly recognized that molecular computers should explore challenging applications unsuitable for existing computers.

In the theory of molecular computing, a variety of computation models have been proposed and investigated. Among others the models of computation based on selfassembly are well recognized to be of great importance in that they can provide one of the most basic

frameworks for molecular computing paradigms. In fact, Adleman's groundbreaking wet lab experiment [1] (solving a small instance of the Hamiltonian Path Problem) is a typical example of molecular computation based on the self-assembly principle.

When we make a brief revisit to the Adleman's original work in 1994, it turns out that his algorithm may be formulated into the schema where component structures are sophisticatedly encoded into linear molecules. From these observations, a general schema of "one pot" selfassembly computation model is outlined as follows:

1. design a finite set of basic units for assembly computation (one may take this finite set as a molecular program),

2. put all those basic units with sufficiently high concentration into one pot, to create a random pool of a single pot (that is, leave the pot for a certain time period, resulting in producing all possible assembly of basic units),

3. (if necessary) perform screening operations to extract only necessary (or unnecessary) assembly of basic units,

4. detect whether or not there is an assembly with desired constraints, then answer yes if there is, and no otherwise.

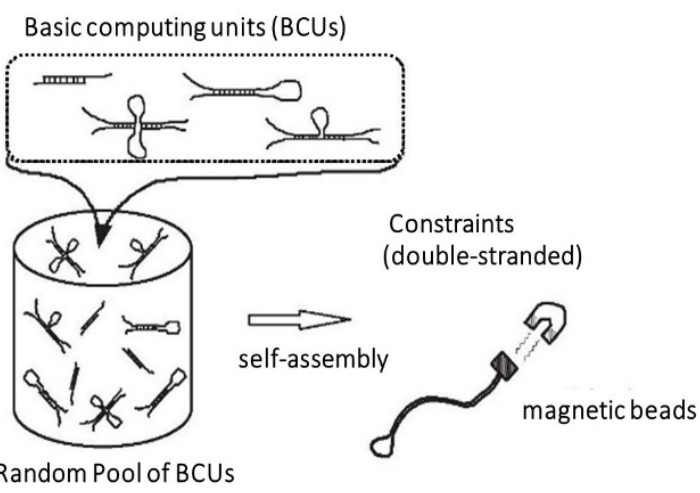

Figure 2. Computation Schema based on Self-assembly

Figure 2 illustrates the overview of computation schema based on self-assembly.

In this section, we will briefly review one of the typical models based on self-assembly computation, called $Y A C$ model. Based on a unique normal form grammar $G$ (proposed by Geffert) that can generate any recursively enumerable language, a computing model YAC has been proposed, in which an input string and every rule in $G$ are translated(encoded) into specific forms of twodimensional structural molecules. These basic blocks (units) of structural molecules with sticky ends are designed so that they may form a DNA complex linearly growing by self-assembly property. This means that YAC performs a simulation of computation (generation) by $G$, where the input string is accepted by YAC if and only if the final DNA complex forms a completely hybridized double-stranded (that is a desired constraint to be checked by screening mechanism).

It is shown that any recursively enumerable language can be recognized by YAC model. Thus, the following holds.

Proposition 1. ([2]) There effectively exists a computing model based on self-assembly principle whose computational power is Turing universal.

Other molecular computing models using highdimensional structures have been proposed to solve NPcomplete problems such as the satisfiability (SAT) problem by Jonoska et al., where 3-dimensional graph structures are used and the algorithm for SAT runs in time proportional to the number variables involved in a given formula. On the other hand, Yokomori investigated a self-assembly computing model in an abstract level, called Computing by Conformational Change (CCC), and proposes the following general schema that

$($ Computation $)=($ Self-Assembly $)+($ Screening Mechanism $)$, where "self-assembly" is due to hybridization of either uncorded or coded molecular components, while "screening mechanism" is regulated by either natural or artificial constraint ([2]). 


\section{Chemical Reaction Paradigm}

Inspired by the work of reaction systems (initiated by [3]), the notion of reaction automata has been introduced in [4] by extending sets in each reaction (of a reaction system) to multisets. We start this section by reviewing basic notions concerning reaction automata.

For a finite set $S$, a reaction in $S$ is a 3-tuple $\mathbf{a}=\left(R_{\mathbf{a}}\right.$, $\left.I_{\mathbf{a}}, P_{\mathbf{a}}\right)$ of finite multisets, such that $R_{\mathbf{a}}, P_{\mathbf{a}} \in S^{\#}, I_{\mathbf{a}} \subseteq S$ and $R_{\mathbf{a}} \cap I_{\mathrm{a}}=\phi$, where $S^{\#}$ denotes the set of all finite multisets over $S$. The multisets $R_{\mathbf{a}}$ and $P_{\mathbf{a}}$ are called the reactant of a and the product of a, respectively, while the set $I_{\mathbf{a}}$ is called the inhibitor of $\mathbf{a}$. These notations are extended to a multiset of reactions. A reaction $\mathbf{a}=\left(R_{\mathbf{a}}, I_{\mathbf{a}}\right.$, $P_{\mathbf{a}}$ ) is applicable to a multiset $D$ if $R_{\mathbf{a}} \subseteq D$ and $I_{\mathbf{a}} \cap D=\phi$. As a result, $D^{\prime}\left(=\left(D-R_{\mathbf{a}}\right) \cup P_{\mathbf{a}}\right)$ is derived.

With a simple example, we introduce the notion of reaction automata. Let us consider a reaction automaton (RA) $\mathcal{A}=\left(S, \Sigma, A, D_{0}, f\right)$ defined as follows:

$S=\left\{p_{0}, p_{1}, a, b, a^{\prime}, f\right\}$ (symbols of objects),

$\Sigma=\{a, b\}$ (input symbols),

$A=\left\{\mathbf{a}_{0}, \mathbf{a}_{1}, \mathbf{a}_{2}, \mathbf{a}_{3}, \mathbf{a}_{4}\right\}$ (reactions) $\}$, where

$\mathbf{a}_{0}=\left(p_{0}, a b a^{\prime}, f\right), \mathbf{a}_{1}=\left(p_{0} a, b, p_{0} a^{\prime}\right), \mathbf{a}_{2}=\left(p_{0} a^{\prime} b, \phi, p_{1}\right)$,

$\mathbf{a}_{3}=\left(p_{1}, a^{\prime} b, a, p_{1}\right), \mathbf{a}_{4}=\left(p_{1}, a b a^{\prime}, f\right)$,

$D_{0}=p_{0}$ (an initial multiset), and $f$ is the final symbol.

Let $w=a a b b$ be a given input string. Since $\mathcal{A}$ has the initial multiset $D_{0}\left(=p_{0}\right)$, there is no reaction in $A$ applicable to $D_{0}$. When receiving the 1 st $a$ of $w$, the multiset $D_{0}$ of $\mathcal{A}$ becomes $p_{0} a$ to which only $\mathbf{a}_{1}$ is applicable and, as a result, $D_{1}\left(=p_{0} a^{\prime}\right)$ is derived. On receiving the 2nd $a$, the multiset of $\mathcal{A}$ becomes $p_{0} a^{\prime} a$ from which $D_{2}\left(=p_{0} a^{\prime} a^{\prime}\right)$ is derived by applying $\mathbf{a}_{1}$. Then, receiving $b$ and applying a lead $D_{2}$ to $D_{3}\left(=p_{1} a^{\prime}\right)$, and on receiving the final $b, \mathcal{A}$ makes $D_{3}$ into $D_{4}\left(=p_{1}\right)$ by a3. After applying $\mathbf{a}_{4}, D_{4}$ eventually leads to the final multiset $f$. Thus, an input string $a a b b$ is accepted by $\mathcal{A}$. This example shows a successful reaction process of $\mathcal{A}$ performed in sequential manner and the set of strings thus accepted by $\mathcal{A}$ is denoted by $L_{\mathrm{sq}}(\mathcal{A})$.

Figure 3 illustrates an overall view of possible reaction processes in $\mathcal{A}$ with inputs $a^{n} b^{n}$ for $n \geqq 0$, and we see that $L_{\mathrm{sq}}(\mathcal{A})=\left\{a^{n} b^{n} \mid n \geqq 0\right\}$ which is a context-free language. We remark that this interactive process can be also performed by $\mathcal{A}$ in maximally parallel manner, i.e. in the manner that every applicable reactions are performed exhaustively. That is, it holds that $L_{\mathrm{mp}}(\mathcal{A})=L_{\mathrm{sq}}(\mathcal{A})$.

Besides both manners of applications in RAs, we often consider an extension of the sequential manner where RA allows a reaction without receiving any input symbol at each step, which is called sequential manner with $\lambda$-input mode.

We investigated the accepting powers of reaction automata with these manners of applying reactions, and obtained the following.

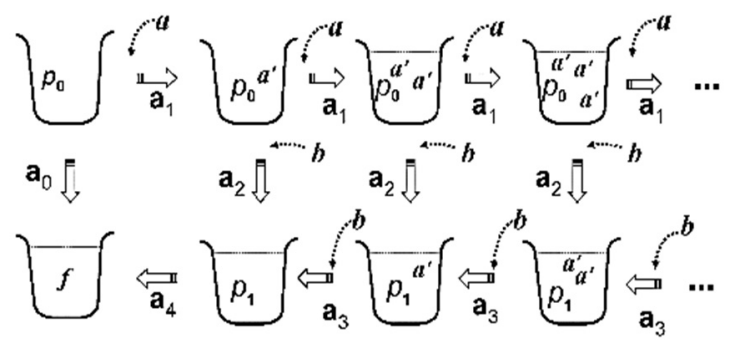

Figure 3. A graphic illustration of interactive processes for accepting strings in $L=\left\{a^{n} b^{n} \mid n \geqq 0\right\}$.

Proposition 2. ([4,5]) The computing power of reaction automata in maximally parallel manner coincides with that of reaction automata in sequential manner with $\lambda$ input mode. Further, both of those computing powers are Turing universal.

On the other hand, the equivalence may not hold for reaction automata in sequential manner with ordinary input mode (i.e., without the use of $\lambda$-input mode).

Proposition 3. ([5]) There exists a recursively enumerable language which cannot be accepted by any reaction automaton in sequential manner.

In order to study the role of inhibitors of reactions in the computing power of RAs, we introduced and studied a restricted RA called Chemical Reaction Automaton (CRA) in which no inhibitor is allowed ([6]).

Further, the space complexity issues of reaction automata (RAs) have been considered. By restricting the volume (i.e., the state of an RA) used in reaction process for a successful computation, some subclasses of RAs were introduced and investigated on relations between classes of languages accepted by those subclasses of RAs and language classes in the Chomsky hierarchy.

Let $f(x)$ be a function defined on the set of natural numbers and $X$ in $\{\mathrm{sq}, \mathrm{mp}\}$. The workspace of $\mathcal{A}$ for $w$ is intuitively defined as the mini-max size of multisets (appearing in all reaction sequences) necessary for accepting $w$. Then, an RA $\mathcal{A}$ is said to be $f(n)$-bounded if for any $w$ in $L_{X}(\mathcal{A})$ with $n=|w|$, the workspace of $\mathcal{A}$ for $w$ is bounded by $f(n)$. Then, four classes of RA languages have been investigated.

Suppose that an RA $\mathcal{A}$ is $f(n)$-bounded. If a function $f(n)$ is a constant $k$ (linear, exponential), then $\mathcal{A}$ is termed constant-(resp. linear-, exponential-) bounded. The class of languages accepted by constant-bounded RAs (linearbounded RAs, exponential-bounded RAs, RAs, CRAs) in $X$ manner is denoted by $\mathcal{C}$ R $\mathcal{A}_{X}$ (resp., $\mathcal{L} \mathcal{R} \mathcal{A}_{X}, \mathcal{E} \mathcal{R} \mathcal{A}_{X}$, $\left.\mathcal{R} \mathcal{A}_{X}, \mathcal{C} \mathcal{R} \mathcal{A}_{X}\right)$. The class of languages accepted by constant-bounded RAs (linear-bounded RAs, exponential-bounded RAs, RAs, CRAs) in $X$ manner with $\lambda$-input mode is denoted by $\mathcal{L} \mathcal{R} \mathcal{A}_{X}^{\lambda}$ (resp., $\mathcal{C}$ o $\mathcal{A}_{X}^{\lambda}$, 


\section{$\left.\mathcal{E} \mathcal{R} \mathcal{A}_{X}^{\lambda}, \mathcal{R} \mathcal{A}_{X}^{\lambda}, \mathcal{C} \mathcal{R} \mathcal{A}_{X}^{\lambda}\right)$}

The results on whole view of language class relations are summarized in Figure 4, where $\mathcal{R E G}(\mathcal{C} \mathcal{F}, \mathcal{C S}, \mathcal{R E})$ denotes the class of regular (resp. context-free, contextsensitive, recursively enumerable) languages in Chomsky hierarchy. Further, $\mathcal{P} \mathcal{N}$ nnotes the class of languages generated by Petri net systems. Thus, except for the class $\mathcal{C F}$, each class in Chomsky hierarchy is exactly characterized by a subclass of RA languages. Further, the following result has been obtained.

Proposition 4. ([6,7]) $A$ language $L$ is in $\mathcal{R E}\left(=\mathcal{R} \mathcal{A}_{m p}\right.$ $=\mathcal{R} \mathcal{A}_{s q}^{\lambda}=\mathcal{C} \mathcal{R} \mathcal{A}_{m p}^{\lambda}$ ) if and only if $L$ is a homomorphic image of some language in $\mathcal{L} \mathcal{R} \mathcal{A}_{m p}$.

\section{Broader Perspective}

Natural computing, (NC) comprises a very broad range of computational principles that varies from wellestablished classical research areas to newly emerging dynamical research areas with great potentiality of many promising applications to interdisciplinary fields.

The former family may include Cellular computation, one of the oldest computing models studied since late 1940s. In Neural computation, artificial neural networks are proposed as computing systems inspired by the biological neural networks mimicking brain functions. Evolutionary computation provides a bunch of algorithms for global optimization strategy inspired by biological evolution. Further, Artificial life is a longlasting research theme from which artificial immune algorithms have been lately developed. As for the latter family of research areas in $\mathrm{NC}$, there are quite a few topics to be mentioned, while due to the space limit we can regretfully name only a part of those emergence of fascinating thoughts here: Membrane computation, Reaction-diffusion computation, Optical computation, Quantum computation, Swarm intelligence, etc.

Further, their applications to practical problems in the real world such as drug discovery in "nano-level engineering" and "medical/life science" are highly recommended to be tackled. For one example, one may refer to a medical application, lately reported, of the use of C.elegans for detecting cancer patients.

Finally, the reader interested in more details about topics discussed here and other many subjects in NC is cordially advised to consult reference papers or appropriate bibliographic sources (such as $[8,9,10])$.

\section{Acknowledgements}

The work partly supported by Partly supported by JSPS KAKENHI, Grant-in-Aid for Scientific Research (C) 17K00021 of The Ministry of Education, Culture, Sports, Science, and Technology, Japan, and by Waseda University grant for Special Research Project: 2017K-121.

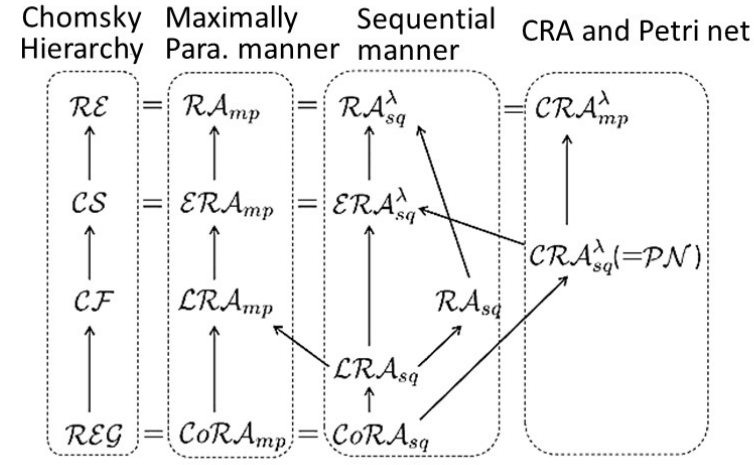

Figure 4. The diagram of the relations among RA language classes and Chomsky hierarchy. (A solid arrow denotes a proper inclusion.)

\section{References}

1. L. Adleman, Molecular Computation of Solutions to Combinatorial Problems, Science, vol.266, pp.1021-1024, 1994.

2. T. Yokomori, Computation $=$ Self-assembly + Conformational Change: Toward New Computing Paradigms, Proc. of 4th International Conference on Developments in Language Theory DLT'99, Aachen, July, pp.21-30, 1999.

3. A. Ehrenfeucht and G. Rozenberg, Reaction systems, Fundamenta Informaticae vol.75, pp.263-280, 2007.

4. F. Okubo, S. Kobayashi and T. Yokomori, Reaction Automata, Theore. Comput. Sci., 429, pp.247-257, 2012.

5. F. Okubo, On the Computational Power of Reaction Automata Working in Sequential Manner, 4th Workshop on Non-Classical Models for Automata and Applications, book@ocg.at series 290, pp.149-164, Osterreichische Computer Gesellschaft, 2012.

6. F. Okubo and T. Yokomori, The Computational Capability of Chemical Reaction Automata, Natural Computing, 15, pp.215-224, 2016.

7. F. Okubo, S. Kobayashi and T. Yokomori, On the Properties of Language Classes Defined by Bounded Reaction Automata, Theore. Comput. Sci., 454, pp.206221, 2012.

8. Gh. Păun, G. Rozenberg, A. Salomaa (Eds.), Handbook of Membrane Computing, Oxford Univ. Press, 2010.

9. G. Rozenberg, T. Bäck and J.N. Kok (eds.), Handbook of Natural Computing, 4 Volumes, Springer, 2012.

10. M.Hagiya and T.Yokomori (eds), Natural Computing Series: Vol.0-Vol.7, Kindai Kagaku-sha, 2011 (in Japanese). 